\title{
ARGUMENTACIÓN EN PREADOLESCENTES: DESCRIPCIÓN DE LA COMPLEJIDAD Y PROFUNDIDAD EN SU ESTRUCTURA DISCURSIVA
}

\author{
ARGUMENTATION IN PRE-ADOLESCENTS: \\ DESCRIPTION OF THE COMPLEXITY AND \\ DEPTH IN THEIR DISCURSIVE STRUCTURE
}

\author{
* \\ Sandra Pérez Pérez Nina Crespo Allende \\ Universidad de Los Lagos, Pontificia Universidad Católica de Valparaíso, \\ Osorno. Valparaíso. \\ sperez@ulagos.cl
}

Entre los diversos modos discursivos, se considera a la argumentación el más complejo y por ello, su adquisición tiene un lugar central en la oralidad tardía, es decir, aquella que tiene lugar durante la edad escolar. Se estima que durante esta etapa, el niño no solo adquiere los elementos de la argumentación, sino que también aprende a complejizarlos para cumplir sus objetivos comunicativos. En este marco, el presente trabajo es un estudio cualitativo de alcance descriptivo que examina la complejidad argumentativa de 18 preadolescentes de 11 años. Los datos se obtuvieron a partir de una tarea de dilema moral que llevó a los niños, organizados en parejas, a resolver una diferencia de opinión en el contexto de una interacción dialógica. El análisis utilizó como fuente la pragmadialéctica, en particular se enfocó en la estructura de la argumentación para observar la interconexión de argumentos al interior del discurso en distintos niveles de incrustación. Nuestros hallazgos revelan que los niños son capaces de construir y organizar un discurso argumentativo complejo. Específicamente, esto se puede visualizar en la explicitud y riqueza de los niveles de sub-argumentación que se manifiesta en su estructura argumental.

Palabras clave: Argumentación infantil, configuración argumental, niveles de subordinación 


\section{2 - Cogency, Journal of reasoning and argumentation}

Among the various discursive modes, argumentation is considered the most complex and, therefore, its acquisition has a central place in late orality, i.e., which takes place during school age. It is estimated that during this stage, the child not only acquires the elements of argumentation, but also learns to make them more complex to meet their communication objectives. In this framework, the present work is a qualitative study of descriptive scope that examines the argumentative complexity of 18 pre-adolescents of 11 years. The data were obtained from a moral dilemma task that led the children, organized in pairs, to resolve a difference of opinion in the context of a dialogic interaction. Pragma-dialectics was used in the analysis, in particular it focused on the structure of the argumentation to observe the interconnection of arguments within the discourse at different levels of embedding. Our findings reveal that children are capable of constructing and organizing complex argumentative discourse. Specifically, this can be seen in the explicitness and richness of the levels of sub-argumentation that is manifested in its argumentative structure.

Keywords: Child argumentation, configuration of argumentative structure, levels of subordination 


\section{Introducción}

Para comprender cabalmente qué implica argumentar, tanto desde un enfoque pragmático como discursivo, es necesario revelar cómo el ser humano aprende a manejar su capacidad argumentativa y, para ello, diversos estudios han centrado su atención en cómo los niños llevan adelante esta modalidad discursiva. Concretamente, en la lengua oral, se ha establecido que los pequeños son capaces de argumentar, ya sea en contextos informales (Boba, 2015; Boba y Arcidiacono, 2014, 2020), como formales (Perret-Clermont, Arcidiacono, Breux, Greco y Miserez-Caperos, 2015) e incluso dando cuenta de procesos inferenciales complejos (Schär, 2017; Greco, Perret-Clermont, Iannaccone, Rocci, Convertini y Schär (2018).

Todos estos estudios nos han dado luces acerca de la complejidad argumentativa de los niños y, en base a estas consideraciones, conviene profundizar en los detalles de esa complejidad, especialmente en la estructura de la argumentación desde un punto de vista más discursivo. En este sentido, dicha estructura deviene relevante no sólo para entender cómo los niños defienden sus puntos de vista, sino también para observar la relación entre los argumentos y sus posibles combinaciones. En este ámbito, van Eemeren y Grootendorst (2006) y van Eemeren y Snoeck Henkemans (2017) señalan que la estructura de la argumentación, presentada por un argumentador en el afán de defender su punto de vista, puede consistir, desde un simple argumento hasta varios argumentos combinados y organizados de distintas maneras. Dentro de sus planteamientos, la complejidad de la estructura de una argumentación no sólo obedece a las características de la disputa que intenta resolver el protagonista, sino también cómo él o ella cree que debería organizar su defensa cada vez que intenta remover las dudas o críticas de su antagonista (van Eemeren et al., 2006). De esta manera, los autores conciben que la complejidad de la argumentación dependerá del número de argumentos únicos que ésta contenga y la relación que existe entre estos argumentos (van Eemeren et al., 2017). En esta misma línea, para Tuzinkievicz, Peralta, Castellaro y Santibáñez (2018), estudiar la complejidad argumentativa de un discurso desde el punto de vista de la pragmadialéctica, permite evaluar la calidad de la misma y su eficacia.

En esta investigación sostenemos que es necesario complementar con otro elemento la comprensión de la estructura argumentativa de la pragmadialéctica, que describe este concepto en términos de cantidad de argumentos únicos y tipos de vínculos establecidos. Consideramos que en esta propuesta se ignora un fenómeno muy interesante en la argumentación y que es especialmente evidente cuando se observa en niños. Los argumentos que clasifica la pragmadialéctica suelen incrustarse unos dentro de otros, de manera similar al modo que operan las estructuras sintácticas cuando se subordinan. Siguiendo la lógica de Givón (2009) para caracterizar dicha subordinación o incrustación de elementos, es posible 
concebir un texto argumentativo como un sistema en el cual los nodos (argumentos únicos) aparecen vinculados en diversas capas o niveles. Mientras más niveles de incrustación tenga una argumentación, podría considerarse más compleja y, por ello, se puede afirmar que el argumentador ha construido un discurso más explícito y sólido para sostener su punto de vista. Así, al incorporar este elemento de análisis para caracterizar el argumento infantil oral, se hace posible ver con más claridad el nivel de dominio que ha alcanzado el niño en la orquestación de su hacer argumentativo.

Por lo tanto, este estudio tiene como objetivo dar cuenta de la estructura argumentativa considerándola como un sistema que se complejiza a partir de la combinación de sus argumentos. La investigación considera un corpus de 18 textos orales producidos por un grupo de niños y niñas de 11 años, en una actividad de argumentación dialógica. Para hacer el análisis, se complementan las herramientas de la pragmadialéctica con el análisis de niveles de subordinación. El desarrollo del trabajo implica, primero, la presentación de los conceptos claves y luego se da cuenta del estudio empírico, su análisis y resultados.

\section{Marco teórico}

\section{La argumentación infantil}

Los estudios de la argumentación infantil, específicamente desde la psicología, han reportado acerca de la dificultad de los niños para producir argumentos y, que solo, ellos podrían ser capaces de argumentar una vez que avanzan en su desarrollo y reciben un entrenamiento adecuado (Kuhn, 1991; Felton y Kuhn, 2001; Goldstein, Crowell y Kuhn, 2009). Contraria a esta postura, otros mencionan que la capacidad de entender y producir argumentos emerge desde muy temprano en los niños (Stein y Albro, 2001; Pontecorvo y Arcidiacono, 2010). Estas diferentes propuestas resultan contradictorias e irreconciliables, y parece ser que los énfasis están más bien puestos en una base individual o en la naturaleza de la interacción social pero los límites claros del fenómeno no quedan en evidencia.

En esta última década, los estudios de argumentación infantil han derivado hacia investigaciones que consideran la argumentación dentro de una discusión crítica (Bova, 2015 Bova et al., 2020; Greco, Mehmeti, Perret-Clermont, 2016, 2017; Schär, 2016, 2017, 2018). Para estos autores, quienes se agrupan en la Escuela de Lugano, la teoría pragmadialéctica ha resultado ser un enfoque significativo para observar el discurso argumentativo de niños en una interacción dialógica entre pares, observando el proceso de argumentación y no ver los argumentos como un producto aislado o cuantificable. Los hallazgos encontrados en estas investigaciones derivan de contextos tanto informales como formales. Dentro de los primeros, se encuentra que los niños son capaces de ofrecer argumentos efectivos para apoyar sus puntos de vista en debates argumentativos relacionados con las reglas y nor- 
mas de los padres. Este aspecto es relevante en términos de las capacidades de los niños para involucrarse en intercambios argumentativos y actuar de manera racional durante la confrontación con sus padres (Bova et al., 2014). Asimismo, se evidencia que los infantes tienden a utilizar los mismos argumentos previamente utilizados por sus progenitores, expresando mayoritariamente argumentos de calidad y cantidad para oponerse a la argumentación con el adulto. Al contrario, cuando los padres presentan argumentos más complejos, tales como aquellos de autoridad, apelación a la coherencia y el argumento de analogía, los niños no avanzan en este tipo de argumentos (Bova, 2015; Bova et al., 2020). En esta misma línea, Schär $(2017,2018)$ declara que los niños pueden involucrarse de manera activa en una disputa argumentativa compleja y, que además, son capaces de abrir nuevos temas de discusión que, igualmente, pueden apoyarlos con uno o dos argumentos.

Ahora bien, en contextos formales como el escolar, los hallazgos indican que niños pre-adolescentes tienen habilidades argumentativas, siendo capaces de iniciar de manera espontánea distintos temas para una discusión argumentativa; entregando sus puntos de vista y apoyándolos con argumentos (Greco et al., 2016). En este sentido, las investigaciones refieren a que los niños pueden seguir a la maestra cuando ella cambia el tema y abren nuevos caminos para una discusión (Greco et al., 2017). Por otro lado, mezclando contextos formales e informales, Perret-Clermont, Schär, Greco, Convertini, Iannaccone y Rocci (2019) concluyen que los niños pueden producir múltiples argumentaciones, y que sus puntos de vista rara vez preexisten a la conversación. Igualmente, ellos son capaces de seguir los temas de una discusión, e incluso presentando sus propios asuntos, a través de sub-discusiones, pero solo si los adultos no los interrumpen.

En referencia a los trabajos mencionados, en el marco de la pragmadialéctica, se ha observado que los niños son capaces de argumentar en contextos tanto formales como informales. No obstante, no existe evidencia de que estos trabajos se hayan centralizado en la complejidad estructural del discurso argumentativo, observando la vinculación entre los argumentos y cómo estos se complejizan, ni tampoco cómo se ordenan en diferentes niveles, que se construyen mientras los niños-argumentadores defienden su posición ante las dudas o críticas de un antagonista. Estos conceptos se desarrollarán de una manera más extensa en el próximo apartado.

\section{La estructura de la argumentación para la pragmadialéctica}

La estructura de la argumentación es fundamental para los enfoques modernos como la teoría pragmadialéctica debido a que es, en principio, necesaria para entender cómo los discutidores defienden sus posiciones y, luego, también indispensable para evaluar la calidad y riqueza de su argumentación (Snoeck Henkemans, 2000). 
En cuanto a los antecedentes vinculados a la estructura argumentativa, ya desde la antigüedad había referencias a la complejidad de esta modalidad discursiva (Snoeck Henkemans, 2000; Plantin, 2018). En el siglo pasado, principalmente desde la lógica informal, Beardsley (1950) fue el primero en representar la estructura argumentativa en un diagrama e introdujo cierta terminología tales como argumentos convergentes, divergentes y de serie. Posteriormente, James Freeman (1992), desde un enfoque funcional, manifestaba que el argumento es básicamente parte de una situación dialéctica (Snoeck Henkemans, 2003) y que la estructura argumentativa debería ser analizada como resultado de un proceso argumentativo (Snoeck Henkemans, 2000). Para este autor (Freeman, 2011) existen tres tipos de estructuras básicas; las de los argumentos convergentes, las de los argumentos ligados y, las de los argumentos en serie (Bermejo Luque, 2011).

Esta última clasificación coincide con la propuesta de la perspectiva pragmadialéctica, la cual considera que la combinación de estas estructuras formaría una argumentación compleja (Snoeck Henkemans, 2000). En una perspectiva similar a la de Freeman, la pragmadialéctica analiza la argumentación considerándola como un diálogo, que busca la resolución de un conflicto. Para este enfoque, poner al descubierto la estructura es importante, porque así se observan -en su externalización- cómo se relacionan estos argumentos y si, en su conjunto, se entiende cómo ellos constituyen una defensa satisfactoria al punto de vista en cuestión (van Eemeren et al., 2017).

Así, la pragmadialéctica distingue entre una estructura simple o compleja. La primera se manifiesta en un argumento único, el cual se compone de una o dos premisas, donde una de ellas usualmente se encuentra implícita (van Eemeren y Grootendorst, 2006).

(1) (a) No es bueno salir a fiestas en el Barrio Sur.

(b) Es peligroso andar de noche.

En (1) se observa, un punto de vista (a) sostenido por un solo argumento (b). El argumentador no puede o no quiere profundizar más su postura y considera que ha sido lo suficientemente explícito.

Por otro lado, la estructura compleja se refiere a una mayor elaboración, y puede ser múltiple, compuesta coordinada o compuesta subordinada. La estructura múltiple (2) refiere a argumentos que - de manera separada - actúan como defensas alternativas al mismo punto de vista (van Eemeren et al., 2006).

(2) (a) En invierno, los osos salvajes no son peligrosos.

(b) Pasan la mayor parte del tiempo durmiendo.

(c) Están muy débiles por falta de comida. 
Zarefsky (2019) plantea que esta forma tiene una estructura que reúne elementos no vinculados, enfatizando la independencia lógica de los diferentes enlaces que van conectando cada argumento (b, c) al punto de vista (a), sugiriendo que se asemeja a un circuito paralelo de electricidad.

Respecto de la estructura compuesta coordinada (3), van Eemeren et al., (2006) mencionan que, en esta forma, cada argumento, de manera individual, se presenta como un apoyo parcial al punto de vista. De este modo, cada elemento, sólo en combinación con otros argumentos, demuestra ser una defensa concluyente.

(3) El rol de la mujer ha cambiado grandemente en los últimos 50 años.

(3a) ahora el cuidado del hogar no es la prioridad

(3 b) es más importante la formación profesional y la independencia económica

Snoeck Henkemans (2003) indica que, en este tipo de estructura, la argumentación consiste en una serie de razones de mayor o menor peso. El protagonista asume que, cada una por separado, no son lo suficientemente convincentes, mientras que pueden ser robustamente concluyentes cuando se toman juntas (Snoeck Henkemans, 2003).

Por último, en la argumentación compuesta subordinada, la defensa del punto de vista se realiza a través de una serie de argumentaciones únicas (4), las cuales se presentan capa por capa y ligadas "de manera vertical", hasta que el punto de vista primario sea defendido de manera concluyente (van Eemeren et al., 2006).

(4) La extinción de las abejas es un tema preocupante

4. a se pone en peligro la vida vegetal del planeta

4. a.1 pues, ellas son las responsables de la polinización

De este modo, van Eemeren y Grootendorst (2006) señalan que varias combinaciones de subargumentaciones, pueden surgir, por ejemplo, "uno de los argumentos que forman parte de una argumentación compuesta coordinada puede ser apoyado por tres subargumentaciones múltiples, y una de las partes constitutivas de una argumentación múltiple puede ser apoyada por dos subargumentaciones compuestas coordinadas" (p.105).

Respecto del fenómeno de subordinación, que también podríamos llamar hasta cierto punto hipotaxis (López García, 1999), en este trabajo se propone incluir el concepto de niveles. Parafraseando el lenguaje de la sintaxis (Mota, 2015; Crespo, Alvarado, Sepúlveda y Figueroa-Leighton, 2019) puede señalarse que, en este tipo de vínculo estructural, existe un elemento (que en este caso es un argumento) subordinado y uno principal o subordinante. Ahora bien, estas construcciones pueden ser recursivas y de alguna manera, un argumento que ya está sosteniendo un punto de vista u otro argumento, puede a su vez, incluir en su 
seno un nuevo argumento subordinado. De esta manera, el texto puede considerarse como articulado en diferentes niveles argumentales que se incluyen unos a otros. Para ilustrar esto se presenta la Figura 1.

PV. La educación no es un lujo, sino una herramienta para ejercer la libertad

A. El que no sabe manejar el código de manera adecuada permanece marginado

B. Además, las exigencias de la actualidad, suponen en los ciudadanos un bagaje de conocimiento común
A1. La sociedad actual está altamente alfabetizada, todo se maneja por escrito

A1.1. No puede acceder a los trámites más sencillos

\begin{tabular}{|l|l|}
\hline $\begin{array}{l}\text { A 1.1.1a } \\
\text { Firmar un } \\
\text { contrato }\end{array}$ & $\begin{array}{l}\text { A 1.1.1b } \\
\text { Sacar un } \\
\text { documento }\end{array}$ \\
\hline
\end{tabular}

Figura 1. Niveles de subordinación

Como se observa en la Figura 1, existe una estructura múltiple, dos argumentos (A) y (B) que sostienen el punto de vista (PV). Además, el argumento (A) subordina a A.1 que la sostiene y la explícita y, a su vez, subordina tres argumentos más que la sustentan. Cada vez que un argumento subordina a otro, lo incorpora a su estructura, es decir, lo incrusta y, por ello, se constituye un nivel más profundo del mismo hilo argumental. Visser (2013) plantea - en una visión dialógica de la argumentación- que esta interconexión de elementos que se sostienen es construida por el argumentador para convencer a su antagonista. $\mathrm{Si}$ comparamos el argumento A y el argumento B que sostienen al punto de vista, quedan en evidencia dos cosas. Por un lado, A incluye más niveles de argumentación en su interior que se han ido subordinando unos a otros y, por otro, que esta estructura es más compleja que la de B y se presenta como más sólida para defender el punto de vista. A partir de allí, es posible ver la estructura de la argumentación, no solo por la modalidad en la que se combinan los argumentos sino por la cantidad de niveles que ellas construyen en su interior. El concepto de cuanto más cantidad de niveles de subordinación incluye un elemento lingüístico, mayor será su complejidad, ha sido propuesto en el ámbito de la sintaxis (Givón, 2009). Sin embargo, la idea puede extenderse y ser muy útil en el caso de la argumentación para poder dar cuenta de manera más precisa del peso de una construcción argumentativa. 
A partir de allí, se propone un análisis que involucre -al describir la estructura argumentalno sólo el tipo de vínculo que establecen los argumentos, sino también el o los niveles de subordinación o incrustación que presentan y dónde se presentan.

\section{Metodología}

\section{Tipo de investigación}

Este trabajo es una investigación de enfoque cualitativo con un alcance descriptivo, cuyo objetivo es dar cuenta de la complejidad de la estructura argumentativa de niños y niñas de 11 años pertenecientes a la Región de Valparaíso.

\section{Sujetos}

Se consideraron 18 niños de 11 años de dos establecimientos subvencionados de la ciudad de Viña del Mar. Específicamente se reclutaron 9 niños y 9 niñas, los cuales se unieron en parejas para llevar a cabo la interacción argumentativa, resultando una conformación de 3 parejas de niñas, 3 parejas de niños y 3 parejas mixtas. Esta propuesta de argumentación entre pares, permite -a nuestro entender- una visión más ecológica del discurso infantil, de cómo este se genera. Sin embargo, como se señala más adelante, existió la figura de un adulto mediador y muchas veces la interacción de los niños se dio con él. El requisito de selección de los sujetos consideró que ellos no deberían tener ningún problema diagnosticado de lenguaje o cognición y deberían cursar el nivel escolar correspondiente para su edad. Cabe señalar, que los padres firmaron un consentimiento informado, autorizando la participación de los sujetos en el estudio y, a la vez, que cada sujeto firmó su asentimiento informado de manera individual y voluntaria.

\section{Instrumento de elicitación}

En este estudio, el objeto de investigación es un corpus verbal que registra un intercambio dialógico de los niños enfrentados a una diferencia de opinión en el marco de un discurso argumentativo entre pares. Para obtener el corpus verbal se utilizó una tarea que contempló un dilema moral denominado 'Robin Hood' (ver anexo 1). Un dilema moral es un texto corto en el cual se presenta una situación problemática que obliga a tomar una postura. Así, cuando el sujeto elige un punto de vista debe fundamentar el por qué y generar una argumentación. El dilema fue presentado por el adulto-investigador, quien, si hacía falta, tomaba el rol de antagonista. Se utilizó este formato siguiendo las propuestas de Schär (2017) y Schär y Greco (2018), quienes han llevado a cabo investigaciones con discurso argumentativo dialógico con niños. Las grabaciones se efectuaron en un espacio conocido para los niños en sus propios establecimientos educacionales. Asimismo, se resguardó que 
dicho espacio fuera lo suficientemente aislado para que los niños pudieran discutir de manera tranquila.

\section{Unidad de análisis}

La unidad de análisis se concentra en los textos orales producidos por cada niño de manera individual, específicamente, se han considerado todos los enunciados que conforman la argumentación de cada sujeto. Estos niños están distribuidos en tres parejas, una de niños, otra de niñas y, además, una de un niño y una niña. Todos los textos fueron analizados sin establecer diferencias de género o de constitución (mixta o no) ya que se consideró que esta es una investigación preliminar. En esto queremos indicar, siguiendo a Greco, PerretClermont, Iannaccone, Rocci, Convertini y Schär (2018), que - a pesar de ver el discurso de cada niño por separado- las construcciones argumentativas de ellos no son consideradas como producciones lingüísticas aisladas para ser evaluadas de manera independiente, ya que son producidas en un contexto de interacción entre niños o entre niños y adulto.

\section{Procedimiento de análisis}

Una vez aplicada la tarea de dilema moral se transcribieron los discursos de los infantes. Luego, a través de una Visión General Analítica de la pragmadialéctica (Palmieri, 2014) se reconstruyó el discurso argumentativo de las parejas, prestando atención a la estructura de la argumentación. Dentro de las distintas configuraciones que presentan las estructuras de argumentos complejos enlazados, que fueron presentados en el marco teórico, fue posible reconocer cinco configuraciones de estructura argumentativa, basadas en la propuesta de van Eemeren y Grootendorst (2004): a. única, b. múltiple, $\boldsymbol{c}$. única-múltiple, $\boldsymbol{d}$. únicacoordinada-múltiple ye. múltiple-coordinada.

Posteriormente, nos enfocamos en analizar los niveles de incrustación que refieren a la subordinación o subargumentación. Para ello, siguiendo la idea general de Givón (2009) sobre jerarquía y complejidad, que ha sido aterrizada a la argumentación por Visser (2013) y Snoeck Henkemans (2003), se considera todo argumento como un sistema, dentro del cual ciertos elementos podrán incluir o subordinar a otros elementos, estableciendo distintos niveles de incrustación. Así, en el contexto de la pragmadialéctica, cada vez que el protagonista reaccione argumentativamente ante las críticas de su antagonista lo conduce a estructuras más complejas de incrustación argumental y, por tanto, genera niveles que permiten visualizar un nuevo aspecto de la complejidad argumentativa.

\section{Análisis de resultados}

El análisis de los datos recolectados permitió observar que los sujetos son capaces de construir y organizar un discurso argumentativo complejo. Específicamente, esto se puede 
visualizar en la explicitud y riqueza de los niveles de subargumentación que se manifiestan en la estructura de dicho discurso. En los datos analizados, se encontraron argumentaciones de distintos niveles en los niños, entendiendo acá que -a partir del segundo nivelse manifiesta la subordinación (o hipotaxis) entre argumentos. En la Figura 2, se ilustran los diferentes niveles de subordinación que manifiesta el discurso de uno de estos niños de 11 años.

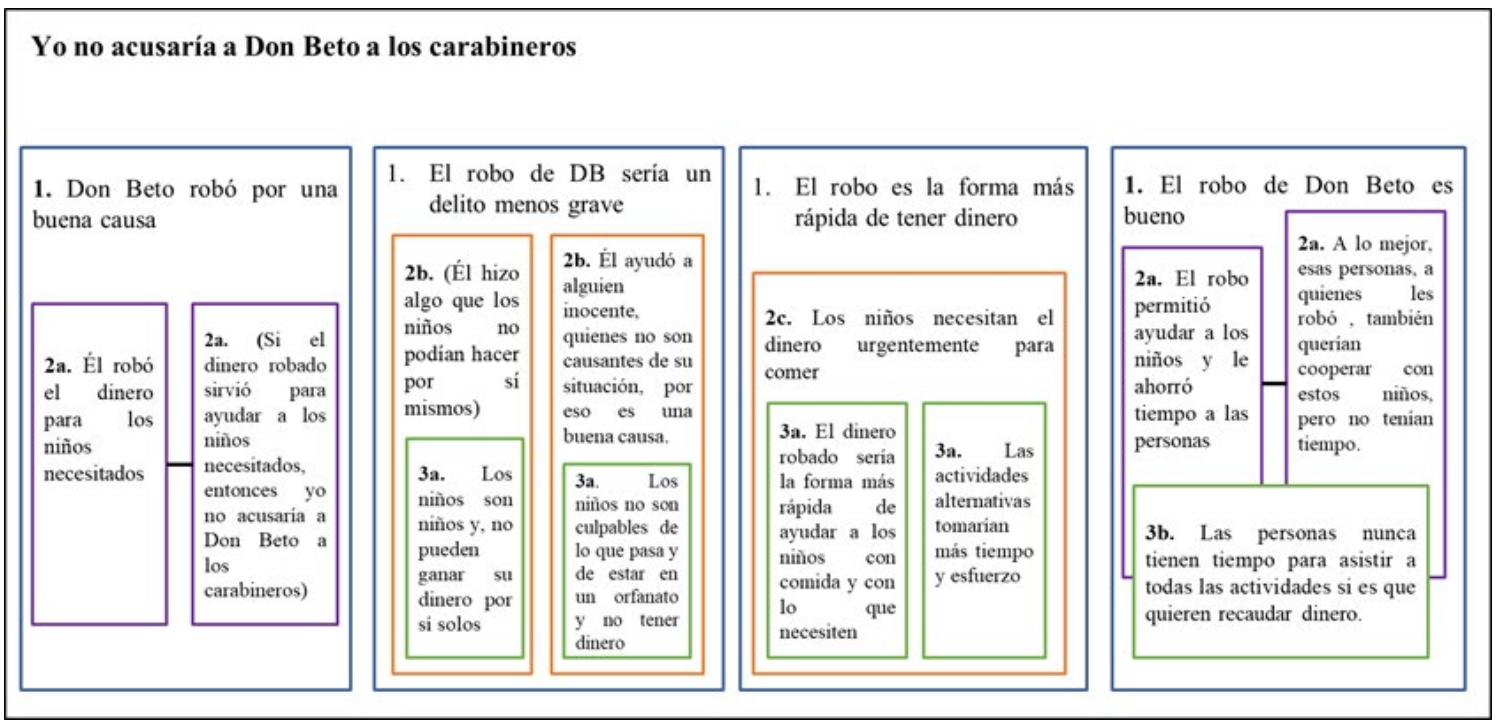

Figura 2. La estructura de la argumentación con tres niveles en una niña

Como puede observarse la estructura presenta 3 niveles de subordinación (representados por los textos enmarcados). En el primer nivel se observa una estructura múltiple, con cuatro argumentos (1). En el segundo nivel, pueden verse diferentes estructuras. Por un lado, se distinguen dos estructuras compuestas coordinadas (2a), por otro, se manifiesta una estructura múltiple con dos argumentos independientes (2b) y, finalmente, un argumento único (2c). Por último, en el tercer nivel se evidencian dos estructuras múltiples (3a) y un argumento único (3b). En este ejemplo aparecen dos elementos de análisis: los niveles de subordinación argumental y la estructura de los argumentos.

Por una parte, los niveles nos señalan el dominio que un sujeto demuestra de su argumentación y cuanto más quiere hacer explícita las razones que sostiene su punto de vista. De esta manera, si el niño hubiera referido sólo el nivel 1 de razones o argumentos señalaría: Yo no acusaría a Don Beto a los carabineros (PV) porque Don Beto robó por una buena causa, el robo sería un delito menos grave, el robo es la forma más rápida de tener dinero, el robo de Don Beto es muy bueno. Existiría una argumentación múltiple, con diversos elementos, que sería válida pero no lo suficientemente explícita (¿Qué es una buena causa? ¿Por qué es un delito menos grave?, etc.). Sin embargo, cuando el niño desarrolla cada uno de sus 
argumentos, va dándole más robustez y sus afirmaciones se vuelven más convincentes. Por ejemplo, en el primer argumento del nivel 1: PV: Yo no acusaría a Don Beto a los carabineros (porque) el robo de Don Beto es un delito menos grave (ya que) él hizo algo que los niños no podían hacer por sí mismos, los niños son niños y, no pueden ganar su dinero por sí solos.

Es importante mencionar que, no todos los sujetos del grupo constituyeron la misma cantidad de niveles ni aportaron el mismo grado de explicitud a sus argumentos. En la Tabla 1 y el Gráfico 1 presentamos la cantidad de niños que construyó un determinado número de niveles y tradujimos ese número al porcentaje relativo.

Tabla 1.

Cantidad y porcentaje de niños que eligen un nivel

\begin{tabular}{|c|c|c|c|c|}
\hline Cantidad niños & 1 & 10 & 6 & 1 \\
\hline Cantidad de niveles & 1 & 2 & 3 & 4 \\
\hline Porcentaje en muestra & 5,5 & 55,6 & 33,4 & 5,5 \\
\hline
\end{tabular}

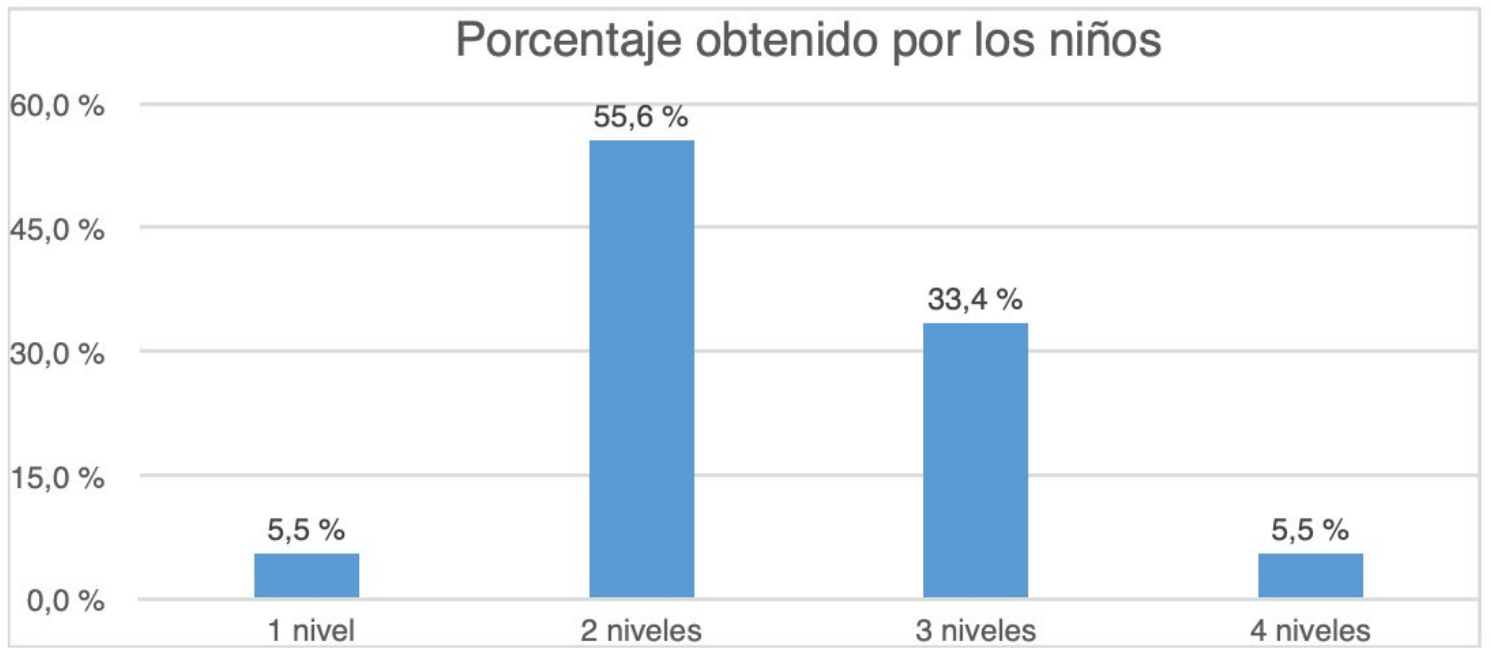

Gráfico 1. Porcentaje obtenidos por niños en cada nivel

Ahora bien, cuando se observan estos datos, es posible señalar que la mayoría de los sujetos prefiere construir usando dos o tres niveles de subordinación en sus argumentos. Así demuestran, por un lado, una conciencia de que deben construir su fundamentación y, por otra, que poseen una habilidad para desenvolver sus argumentaciones en niveles subordinados. No está al alcance de este estudio señalar cómo se da esta elaboración en niños menores o mayores que los de la muestra, pero es muy probable que este sea un logro del desarrollo tardío del lenguaje (Berman, 2004) muy propio en la pre-adolescencia. Siendo así, se hace evidente la voluntad de participar en una disputa dialógica para convencer a su oponente a través de la palabra. El número de niveles de subordinación revelaría una orquestación de los recursos argumentativos con un fin comunicativo determinado, el dominio no es sólo 
lingüístico estructural, sino pragmático porque implica un manejo del lenguaje adecuándose a las demandas de la interacción argumentativa. En este sentido, lo pragmático está vinculado a la búsqueda de la persuasión de la persona a la cual va dirigido el discurso (van Eemeren y Houtlosser, 2002)

Por otra parte, las estructuras ya señaladas por van Eemeren y Snoeck Henkemans (2017) parecieran funcionar en el discurso argumentativo de estos niños como una manera de enlazar los elementos que van configurando en él. En este trabajo se ha dejado de lado la figura de subordinación porque está descrita en los niveles. A partir de allí, se han reconocido en el corpus -considerando la propuesta de la pragmadialéctica- cinco categorías para describir cómo se combinan las estructuras al interior de un nivel, conformando lo que se ha decidido denominar configuraciones argumentativas (Ver Procedimiento de Análisis). $\mathrm{Al}$ analizar los discursos de los niños fue posible determinar la presencia de 43 de estas configuraciones en todo el corpus. En la Tabla 2 y el Gráfico 2, se presenta la cantidad de configuraciones construidas y su peso porcentual en el total de realizaciones

Tabla 2.

Cantidad y porcentaje de configuraciones argumentativas en el corpus

\begin{tabular}{|c|c|c|c|c|c|}
\hline & A) ÚNICA & B) MÚLTIPLE & $\begin{array}{c}\text { C) ÚNICA Y } \\
\text { MÚLTIPLE }\end{array}$ & $\begin{array}{c}\text { D) ÚNICA, COORDI- } \\
\text { NADA Y MÚLTIPLE }\end{array}$ & $\begin{array}{c}\text { E) MÚLTIPLE Y } \\
\text { COORDINADA }\end{array}$ \\
\hline $\begin{array}{c}\text { Cantidad de } \\
\text { configuraciones }\end{array}$ & 12 & 21 & 6 & 2 & 2 \\
\hline Porcentaje & 27,9 & 48,7 & 14 & 4,7 & 4,7 \\
\hline
\end{tabular}

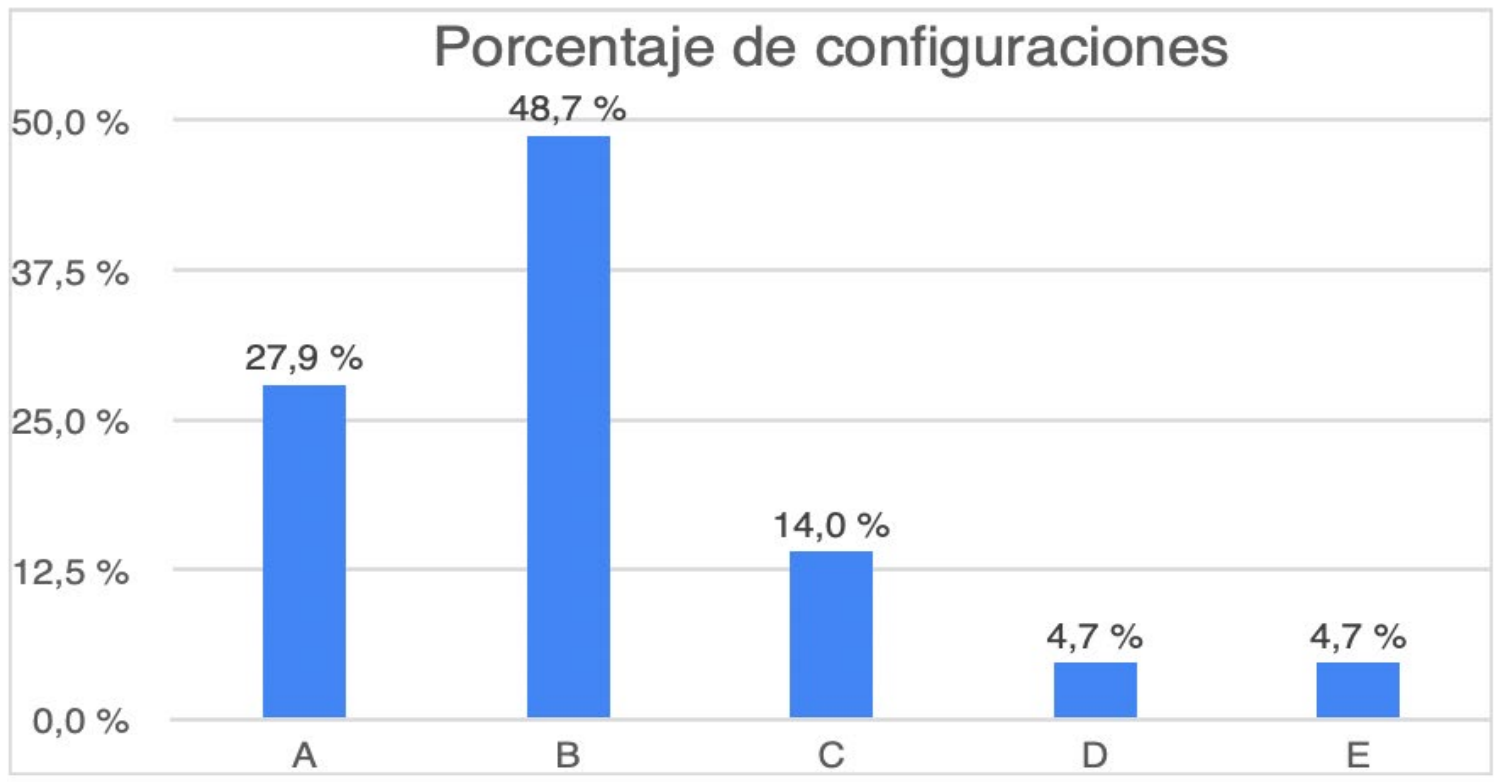

Gráfico 2. Porcentaje de configuraciones 
Cuando se observan estos datos, es posible indicar que la mayoría de los argumentos observados fueron múltiples, es decir, series de argumentos únicos yuxtapuestos que no se conectan entre sí. En su defecto se caracterizan por realizar argumentos únicos o establecer combinaciones de múltiples y únicos en un nivel. Las configuraciones que incluyen elementos coordinados (es decir vinculados entre sí) son menos frecuentes e incluso en esta edad, los elementos coordinados no aparecen configurando un nivel por sí mismos. ¿Por qué ocurre esto? recordemos que en la estructura coordinada, los dos elementos cooperan entre sí para robustecer un argumento que apoya el punto de vista defendido, a los 11 años esta vinculación más micro entre elementos no parece estar totalmente lograda, sin embargo, para poder entender el fenómeno de manera completa habría que tener datos de edades mayores y ver cómo opera esta lógica.

\section{Conclusiones}

Este estudio tuvo como objetivo dar cuenta de la estructura argumentativa considerándola como un sistema que se complejiza a partir de la combinación de sus argumentos (Snoeck Henkemans, 2000, 2003; van Eemeren et al., 2017). El resultado nos ha permitido observar, contrario a los estudios que planteaban acerca de la dificultad de los niños para argumentar (Felton et al., 2001; Goldstein et al., 2009), que a los 11 años, ellos son capaces de organizar y construir un discurso argumentativo complejo.

En cuanto al análisis, aparecen dos elementos esenciales, los niveles de subordinación argumental y la estructura de los argumentos. En lo que respecta al primero, los hallazgos muestran que existe una diversidad en la construcción de niveles de subordinación entre los niños, algunos presentan un solo nivel y otros, en cambio, llegan a construir cuatro niveles de subordinación. Sin embargo, la muestra indica que los niños mayoritariamente construyen utilizando dos o tres niveles de subordinación en sus discursos. Lo anterior refleja que los preadolescentes a los 11 años ya demuestran tener conciencia de estar dentro de una discusión argumentativa que requiere, no solo brindar un punto de vista, sino que están obligados a defenderlo y fundamentarlo para lograr su objetivo como argumentadores. Además, se agrega en estos hallazgos un nuevo elemento, los niños no solo complejizan su hacer en el plano de los contenidos, sino que son capaces de armar niveles estructurales de argumentos interrelacionados, completando sistemas más o menos complejos que les permiten lograr una mayor explicitud para convencer a su antagonista. Lo anterior coincide con estudios que refieren a que los niños son capaces de ofrecer argumentos efectivos para apoyar sus puntos de vista (Bova, 2015) y que tienen la habilidad para involucrarse en intercambios argumentativos en una confrontación con otros (Bova et al., 2014). 
En relación a la estructura, y específicamente, a las configuraciones argumentativas encontradas al interior de cada nivel, los hallazgos evidencian que la mayoría de los sujetos prefiere utilizar estructuras múltiples, es decir, una serie de argumentos únicos que apoyan al punto de vista primario u otro argumento dentro de un nivel construido. Concretamente, los niños realizan configuraciones con argumentos únicos y que también establecen combinaciones múltiples y únicas en dichos niveles. En un estudio con infantes de 2 a 6 años, Perret- Clermont et al. (2019), ya mencionaban que los niños, en interacciones argumentativas, eran capaces de producir argumentaciones múltiples. Nuestro estudio, con preadolescentes nos ha permitido demostrar que en esta edad, ellos continúan prefiriendo argumentos múltiples pero avanzan en la construcción de una variedad de estructuras combinándolos con argumentos únicos y coordinados.

En el plano teórico, este estudio evidenció la utilidad de complementar la noción de estructura argumentativa de la pragmadialéctica (van Eemeren et al., 2017) con el concepto de niveles de subordinación argumental, adaptados de propuestas sintácticas (Givón, 2009). Consideramos que esta combinación permite hacer más transparente la descripción de la construcción del discurso argumentativo para lograr objetivos retóricos. Por ello, la propuesta puede resultar una herramienta muy poderosa para dar cuenta de cómo esta habilidad se desarrolla en los niños y jóvenes hispanohablantes que son puestos en la tarea de resolver una diferencia de opinión. Dentro de la corriente funcionalista de Berman (2004), el dominio lingüístico de una modalidad discursiva está indudablemente unido al manejo estratégico de elementos, que tiene que ver con su complejidad estructural. Sin duda, el incremento en el léxico puede estar también implicado, pero el énfasis en lo estructural, que es lingüístico, pues se relaciona con mecanismos similares a los que gobiernan la subordinación (como fue señalada en el marco teórico), parece ser lo más característico.

Finalmente, cabe señalar la importancia que reviste el discurso argumentativo, como una herramienta dialéctica que desarrolla el pensamiento crítico (Alzate, 2012); y que -a su vez- evidencia un dominio lingüístico discursivo más acabado por parte de los hablantes de una lengua (Nir y Berman, 2010). Por todo ello, creemos que esta investigación podría continuarse, considerando niños de otras edades (menores y mayores) o de otras condiciones (de desarrollo típico o atípico, hablantes de L1 y L2), para observar cómo se produce el desenvolvimiento de esta modalidad lingüística tan valiosa para el ser humano y su participación en el mundo social. 


\section{Referencias}

Alzate, O. E. T. (2012). La argumentación como constituyente del pensamiento crítico en niños. Hallazgos, 9(17), 211-233.

Bermejo Luque, L. (2011). Reseña: James B. Freeman Argumentation Structure: Representation and Theory. Revista Iberoamericana de Argumentación, (3), 1-7.

Berman, R. (Ed.). (2004). Language development across childhood and adolescence. John Benjamins Publishing.

Beardsley, M. (1950). Practical logic. New York: Prentice Hall

Bova, A., y Arcidiacono, F. (2020). Las respuestas de los niños en discusiones argumentativas relacionadas con las reglas y normas de los padres. Revista signos, 53 (102), 8-30.

Bova, A. (2015). Children's responses in argumentative discussions relating to parental rules and prescriptions. Ampersand, 2, 109-121.

Bova, A., y Arcidiacono, F. (2014). You must eat the salad because it is nutritious". Argumentative strategies adopted by parents and children in food-related discussions at mealtimes. Appetite, 73, 81-94.

Crespo, N., Alvarado, C., Sepúlveda, J., y Figueroa-Leighton, A. (2020). El desarrollo típico y atípico de la subordinación en español. Estudio en dos poblaciónes. Literatura $y$ lingüística, (41), 353-381.

Felton, M., y Kuhn, D. (2001). The development of argumentative discourse skill. Discourse processes, 32(2-3), 135-153.

Freeman, J. B. (2011). Argument Structure: Representation and Theory. New York: Springer

Freeman, J. B. (1992). Dialectics and the Macrostructures of Arguments: A Theory of Argument Structures. Berlin: De Gruyter

Givón, T. (2009). The Genesis of Syntactic Complexity. Amsterdam: John Benjamin Publishing Company.

Goldstein, M., Crowell, A., y Kuhn, D. (2009). What constitutes skilled argumentation and how does it develop?. Informal Logic, 29(4), 379-395. 
Greco, S., Perret-Clermont, A-N., Iannaccone, A., Rocci, A., Convertini, J. y Shär, R. (2018). The Analysis of Implicit Premises within Children's Argumentative Inferences. Informal Logic 38 (1), 438-470.

Greco, S., Mehmeti, T., y Perret-Clermont, A. N. (2017). Do adult-children dialogical interactions leave space for a full development of argumentation?: A case study. Journal of Argumentation in Context, 6(2), 193-219.

Greco Morasso, S., Mehmeti, T., y Perret-Clermont, A. N. (2016). Getting involved in an argumentation in class as a pragmatic move: Social conditions and affordances. En Argumentation and Reasoned Action: Proceedings of the First European Conference on Argumentation. (Vol. 2, pp. 463-478). College Publications.

Kuhn, D. (1991). The skills of argument. Cambridge University Press.

López García, A. (1999). Relaciones paratácticas e hipotácticas. En Bosque, I. y Demonte, V. (Ed.), Gramática descriptiva de la lengua española (pp. 3507-3548). Madrid: Espasa Calpe, S.A.

Mota, S. (2015). ¿Por qué se usa 'recursión' cuando se quiere significar auto-inclusión?: Clarificaciones conceptuales sobre la recursión en el programa Chomskiano. RLA. Revista de Lingüística Teórica y Aplicada, 53(2), 171-191.

Nir, B., Berman, R. A. (2010). Complex syntax as a window on contrastive rhetoric. Journal of Pragmatics, 42(3), 744-765.

Palmieri, R. (2014). Corporate argumentation in takeover bids. John Benjamins Publishing Company.

Perret-Clermont, A. N., Schär, R. G., Greco, S., Convertini, J., Iannaccone, A., y Rocci, A. (2019). Shifting from a monological to a dialogical perspective on children's argumentation. Lessons learned. En F, H. van Eemeren y B. Barssen. (Ed.), Argumentation in actual practice. Topical studies about argumentative discourse in context (pp.211-236) Ámsterdam: John Benjamins Publishing Company.

Perret-Clermont, A-N., Arcidiacono, F., Breux S., Greco, S. y Miserez-Caperos, C. (2015). Knowledge-oriented argumentation in children. En F.H. van Eemeren y B. Garssen (Ed.), Scrutinizing Argumentation in Practice (pp.135-150). Ámsterdam: John Benjamins Publishing Company. 
Pontecorvo, C., Arcidiacono, F. (2010). Development of reasoning through arguing in young children. Cultural-Historical Psychology, (4).

Plantin, C. (2018). Dictionary of Argumentation. An Introduction to Argumentation Studies. Milton Keynes, UK: College Publications.

Schär, R. (2018). On the negotiation of the issue in discussions among young children and their parents. Revue Tranel, 68, 17-25.

Schär, R., Greco, S. (2018). The emergence of issues in everyday discussions between adults and children. International Journal of Semiotics and Visual Rhetoric, 2(1), 29-43.

Schär, R. (2017). Definitional arguments in children's speech. Lanalisi linguistica e letteraria 25 (1), 173-192.

Schär, R. (2016). Uses of arguments from definition in children's argumentation. En Argumentation, Objectivity, and Bias: Proceedings of the 11th International Conference of the Ontario Society for the Study of Argumentation (pp. 1-15). Canada: OSSA.

Snoeck Henkemans, F. (2000). State-of-the-art: The structure of argumentation. Argumentation, 14(4), 447-473.

Snoeck Henkemans, F. (2003). Complex argumentation in a critical discussion. Argumentation, 17(4), 405-419.

Stein, N. L., Albro, E. R. (2001). The origins and nature of arguments: Studies in conflict understanding, emotion, and negotiation. Discourse processes, 32(2-3), 113-133.

Tuzinkievicz, M. A., Peralta, N., Castellaro, M. y Santibáñez, C. (2018). Complejidad argumentativa individual escrita en estudiantes universitarios ingresantes y avanzados. Liberabit 24 (2), 231-247.

van Eemeren, F. H. y Snoeck Henkemans, F. (2017). Argumentation: Analysis and Evaluation (2da ed.). New York: Taylor \& Francis.

van Eemeren, F. H. y Grootendorst, R. (2006). Argumentación, Comunicación y Falacias: Una perspectiva pragma-dialéctica. Segunda Edición en Chile. Ediciones Universidad Católica de Chile. 
van Eemeren, F.H.,y Grootendorst, R. (2004). A systematic theory of argumentation: The pragma-dialectical approach. Cambridge University Press.

van Eemeren, F.H. \& Houtlosser, P. (2002). Strategic maneuvering: Maintaining a delicate balance. In van Eemeren \& Houtlosser (Eds.), Dialectics and rhetoric: The warp and woof of argumentation analysis (pp. 131-159). Dordrecht: Kluwer Academic Publishers.

Visser, J. (2013). A formal account of complex argumentation in a critical discussion. En Virtues of argumentation: proceedings of the 10th International Conference of the Ontario Society for the Study of Argumentation (OSSA), 22-26 May 2013 University of Windsor.

Zarefsky, D. (2019). The Practice of Argumentation: Effective Reasoning in Communication. Cambridge University Press 